\title{
The Effect of Money Priming on Self-Focus in the Imitation- Inhibition Task
}

\section{A Registered Report}

\author{
Oliver Genschow ${ }^{1}$, Johannes Schuler ${ }^{2}$, Emiel Cracco ${ }^{3}$, Marcel Brass ${ }^{3}$, and Michaela Wänke ${ }^{4}$ \\ ${ }^{1}$ Faculty of Human Sciences, Social Cognition Center Cologne, University of Cologne, Germany \\ ${ }^{2}$ Fraunhofer Institute for System and Innovation Research, Germany \\ ${ }^{3}$ Department of Experimental Psychology, Faculty of Psychology and Educational Sciences, Ghent University, Belgium \\ ${ }^{4}$ Department of Experimental Psychology, Faculty of Psychology and Educational Sciences, University of Mannheim, Germany
}

\begin{abstract}
The self-sufficiency hypothesis suggests that priming individuals with money makes them focus more strongly on themselves than on others. However, recently, research supporting this claim has been heavily criticized and some attempts to replicate have failed. A reason for the inconsistent findings in the field may lay in the common use of explicit measures, because they tend to rely on one or just a few items and are thus prone to demand effects and low reliability. In the present research, we administered, in two experiments, the imitation-inhibition task - a robust, unobtrusive, and reliable paradigm that is sensitive to self-other focus on a trial-by-trial basis. A pilot study found an increased focus on the self as compared to others when primed with money. Building on this finding, a preregistered high-powered experiment replicated this effect, suggesting that money primes may indeed increase a focus on the self. An additionally carried out meta-analysis indicates that automatic imitation is modulated by self-other focus and that money primes lead to a smaller focus on the self than conventional methods. Overall, the found effects are rather small and several limitations, such as order effects, call for a cautious interpretation of the findings.
\end{abstract}

Keywords: money priming, automatic imitation, self-other focus

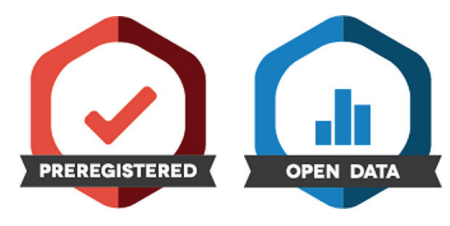

Based on seminal findings in the domain of money priming, Vohs, Mead, and Goode (2006) introduced more than a decade ago the self-sufficiency hypothesis, according to which people who are reminded of money evaluate options and actions in terms of personal inputs and outputs. This general market pricing orientation (Fiske, 1991) is accompanied by an inner state of self-sufficiency "wherein people put forth effort to attain personal goals and prefer to be separate from others" (Vohs et al., 2006, p. 1154). As a consequence, people focus more strongly on self-related needs and less on the needs of others (Mok \& De Cremer, 2018; Reutner \& Wänke, 2013; Schuler \& Wänke, 2016; Vohs, Mead, \& Goode, 2008).

A series of findings supports the theoretical consideration that priming people with money increases a focus on the self (e.g., Gasiorowska, Zaleskiewicz, \& Wygrab, 2012; Vohs et al., 2006; for a review see Vohs, 2015). For example, when being primed with money, participants show an increased focus on one's own benefits and goals (Gąsiorowska \& Hełka, 2012; Reutner \& Wänke, 2013; Teng, Chen, Poon, Zhang, \& Jiang, 2016) and put more emphasis on the achievement of personal tasks (Boucher \& Kofos, 2012; Zedelius, Veling, \& Aarts, 2013). Likewise, it has been found that subjects reminded of money are less inclined to interact with others (Vohs et al., 2006), to help others (e.g., Guéguen \& Jacob, 2013; Vohs et al., 2006) and to consider other persons' needs (Gino \& Mogilner, 2014; Kouchaki, Smith-Crowe, Brief, \& Sousa, 2013).

Despite the support for the idea that money priming results in a focus on the self, such research has recently undergone heavy criticism. More specifically, several money priming studies did not replicate as expected (Klein et al., 2014; Rohrer, Pashler, \& Harris, 2015; Schuler \& Wänke, 2016) and two articles were suspected (one convicted) of data fraud (for details, see Pashler, Rohrer, Abramson, Wolfson, \& Harris, 2016). Even more concerning, a 
meta-analysis by Vadillo, Hardwicke, and Shanks (2016) suggests that the seminal findings on money priming that inspired the entire field (i.e., Vohs et al., 2006) might have been biased by selective reporting and other questionable research practices (see also Lodder, Ong, Grasman, \& Wicherts, 2019). Moreover, in a recent effort to shed light onto these mixed effects, Caruso, Shapira, and Landy (2017) investigated, across three high-powered studies $(N=4,649)$, the degree to which different money priming manipulations affect different self-other focus related selfreports. The results indicated that the effects of money priming are rather weak and inconsistent across measures and studies.

Together, these research findings raise the question why money priming manipulations produce such inconsistent findings. A possible answer may lay in the used methods. That is, previous research on money priming has mostly assessed behavioral measures based on one or just a few items, which is known to result in low reliability (cf. Epstein, 1980). Moreover, most studies used explicit self-reports that may be prone to demand effects (e.g., Schwarz, 1999). Finally, in many studies there was a time delay between the prime and the dependent variable, which may reduce the strength of the effect (e.g., Hermans, De Houwer, \& Eelen, 2001; Hermans, Spruyt, \& Eelen, 2003).

These shortcomings in measuring the effects of money priming call for a new methodological approach. Therefore, in the present research we aimed to test the core assumption underlying the self-sufficiency hypothesis (i.e., money primes lead to an increased focus on the self; Vohs et al., 2006) with a highly reliable task that has been demonstrated to be sensitive to self-other focus and is rather unobtrusive. Moreover, we integrated the money primes within each trial to strengthen the effect of the manipulation. A promising task that allows for all these characteristics is the imitation-inhibition task (Brass, Bekkering, Wohlschläger, \& Prinz, 2000; Stürmer, Aschersleben, \& Prinz, 2000), which is typically used in the study of automatic imitation (for a meta-analysis see Cracco, Bardi, et al., 2018).

\section{Automatic Imitation}

Individuals imitate a wide range of different behaviors including postures (LaFrance, 1982), gestures (Cracco, Genschow, Radkova, \& Brass, 2018), or simple movements (Genschow \& Florack, 2014; Genschow, Florack, \& Wänke, 2013; Genschow \& Schindler, 2016). According to ideomotor theory (e.g., Greenwald, 1970; Prinz, 1990, 1997), such imitative tendencies are the result of shared perception-action codes that cause observed actions to be represented in the same format as executed actions. These shared representations provide a common ground for information on the self and others, suggesting an intrinsic overlap between self and other in the brain (e.g., Lamm, Bukowski, \& Silani, 2016).

The most prominent task in the domain of automatic imitation is the imitation-inhibition task (Brass, Bekkering, \& Prinz, 2001; Brass et al., 2000). In this task, participants respond, across many trials, to symbolic stimuli by lifting 1 of 2 fingers. For example, participants have to lift their index finger in response to the number " 1 " and their middle finger in response to the number " 2 ". Together with the presentation of the number on the screen, participants see another person's hand either lifting the same finger (congruent trial) or the other finger (incongruent trial). Past research has shown that congruent finger lifting movements speed up responses and incongruent finger lifting movements slow down responses (Brass et al., 2001, 2000). The difference in response speed between incongruent and congruent trials is the imitation-inhibition congruency effect, which reflects the degree of confusion between internal and external action plans and as such can be seen as a measure of self-other focus (Brass, Ruby, \& Spengler, 2009).

Indeed, research has shown that an increased focus on the self as compared to others reduces the congruency effect (Cracco, Clauwaert, Van den Broeck, Van Damme, \& Brass, 2019; Hogeveen \& Obhi, 2011; Leighton, Bird, Orsini, \& Heyes, 2010; Spengler, Brass, Kühn, \& Schütz-Bosbach, 2010; van Baaren, Maddux, Chartrand, De Bouter, \& van Knippenberg, 2003; Wang \& Hamilton, 2013). For instance, Hogeveen and Obhi (2011) primed participants with words related to an interdependent self-construal or independent self-construal and found smaller congruency effects during independent as compared to interdependent primes. Relatedly, Spengler and colleagues (2010) found reduced congruency effects when increasing participants' self-focus by letting them sit in front of a mirror during the imitationinhibition task.

In sum, the imitation-inhibition task has been demonstrated to be sensitive to self-other focus. Moreover, the task is known to be reliable and robust. For example, in a recent highly powered study $(N=196)$, Genschow et al. (2017) found a split-half reliability of $\rho^{*}=.86$. Moreover, a recent meta-analysis on the imitation-inhibition task yielded an effect size of $g_{z}=1.03$ for reaction times, indicating a very strong effect (Cracco, Bardi, et al., 2018).

\section{Present Research}

The aim of the present research was to administer a robust, unobtrusive and reliable paradigm that is sensitive to selfother focus in order to test the influence of money priming on self-focus. We applied the imitation-inhibition task and hypothesized smaller congruency effects during trials that 
contain money primes as compared to control primes. We first ran a pilot study. Based on the results of the pilot study we adapted our paradigm and then ran a highly powered preregistered experiment. Stimuli and data of both experiments, and the preregistration of the preregistered experiment are made open accessible at the Open Science Framework (OSF) under the following link: https://osf.io/ ncvfs/?view_only=82162828bf194135b8bb63b483ed2870

\section{Pilot Study}

\section{Method}

\section{Participants and Design}

In return for partial course credit, 47 students from Ghent University (Belgium) participated in this study. All participants reported normal or corrected-to-normal vision. Prior to analysis, we excluded five participants: Two participants were excluded because the response box did not detect any response and three participants were excluded because they committed errors in more than $50 \%$ of the trials, suggesting random responses. The final sample thus contained 42 participants (12 males), with an age ranging from 18 to $27(M=21.90 ; S D=2.97)$. The design consisted of a 2 (Congruency: congruent trials vs. incongruent trials) $\times 2$ (Priming: money vs. control) within-subject design.

\section{Stimuli and Apparatus}

The imitation-inhibition task (Brass et al., 2001, 2000) was programmed using Tscope5 software (Stevens, Lammertyn, Verbruggen, \& Vandierendonck, 2006) and was run on Asus Eee PC $1215 \mathrm{~N}$ laptops with an external 17" Dell monitor. Responses were recorded with a custom-built response box, which uses light sensors to detect lifting movements of participants' index and middle fingers. Stimuli consisted of different frames $(523 \times 422$ pixels $)$. The frames depicted a female left hand that was positioned to create a mirror image of the participant's right hand (i.e., the response hand). To produce an illusion of movement, the hand was first presented in resting position and was then overwritten by a second picture of the hand in the final position. The final position depicted the model either having the index finger or the middle finger lifted. Depending on condition, the model's hand was either lying on a bunch of money bills (i.e., $100 €, 20 €, 10 €$ bills) or on paper sheets that contained the exact same pixels as the money bills, but were regrouped in random order.

\section{Procedure}

The pilot study was conducted at the end of a series of other experiments. After participants signed a written informed consent, the experimenter gave some general oral

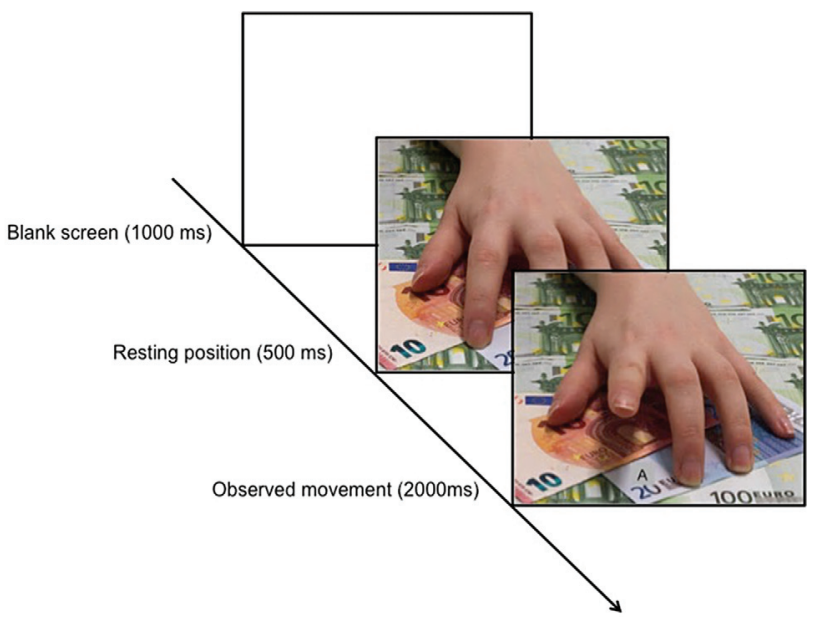

Figure 1. Procedure of a trial in the imitation-inhibition task.

instructions on the imitation-inhibition task. Participants then ran through eight practice trials to get familiar with the task. Afterward, participants performed two blocks of the imitation-inhibition task. In one block participants saw a hand lying on money bills (see Figure 1) and in the other block they saw the same hand lying on paper sheets. Within each block, the background (i.e., money bills or paper sheets) was always the same. The reason of using a blocked manipulation instead of a trial-by-trial approach was to reduce the possibility of carry over effects. Moreover, as further protection against carry over effects, we introduced a short break between the two blocks. The order of the experimental blocks was counterbalanced across participants and each block consisted of 120 trials. After the experiment participants were fully debriefed and dismissed.

\section{Imitation-Inhibition Task}

Participants had to lift their index or middle finger in response to a letter $(\mathrm{A}=$ lift index finger, $\mathrm{B}=$ lift middle finger) on the computer screen while watching a congruent or incongruent finger movement (cf. Figure 1). Congruent and incongruent trials were presented randomly. Each trial started with the appearance of a picture showing a female hand mirroring participants' right hand in resting position for $500 \mathrm{~ms}$. Afterward, a second picture of the model lifting either the index or middle finger was presented for 2,000 ms or until participants responded. Simultaneously, the imperative cue (i.e., letter A or B) was displayed between the model's index and middle finger. Between trials, the screen remained blank for $1000 \mathrm{~ms}$.

\section{Data Preparation}

To prepare data for analysis, we excluded excessively fast and slow trials. That is, trials with a reaction time (RT) faster than $100 \mathrm{~ms}(0.02 \%)$ and trials with a RT slower than $1,000 \mathrm{~ms}(0.63 \%)$ were excluded (cf. Catmur \& 
Heyes, 2011; Cracco, De Coster, Andres, \& Brass, 2015). For the RT analyses, erroneous trials (7.42\%) were excluded as well.

\section{Reliability}

In order to test the reliability of the imitation-inhibition task we assessed the split-half reliability. First, we computed the congruency effect for even trials and for odd trials and then computed the Spearman-Brown coefficient. The results yielded $\rho^{*}=.70$ for the congruency effect.

\section{Results}

\section{Latencies}

We hypothesized smaller congruency effects in the money priming condition as compared to the control condition. To test this hypothesis, we conducted a 2 (Congruency: congruent trials vs. incongruent trials) $\times 2$ (Priming: money vs. control) ANOVA for repeated measures. The results yielded a main effect for congruency, $F(1,41)=128.37$, $p<.001, d_{\mathrm{z}}=1.75$, indicating that participants responded faster (measured in ms) in congruent trials $(M=410.68$; $S D=23.97)$ than in incongruent trials $(M=447.32 ; S D=$ 43.85). The main effect for priming was not significant, $F(1,41)=0.28, p=.60$. More important for our predictions, however, was the interaction between congruency and priming, $F(1,41)=4.09, p=.050, d_{\mathrm{z}}=.31$, indicating smaller congruency effects in the money priming condition $(\Delta=32.69 ; S D=25.76)$ than in the control condition $(\Delta=$ 40.58; $S D=23.12)$. The results are depicted in Figure 2 .

\section{Additional Analyses}

In additional analyses, we first analyzed the error rates of the imitation-inhibition task with a 2 (Congruency: congruent trials vs. incongruent trials) $\times 2$ (Priming: money vs. control) ANOVA for repeated measures. The ANOVA yielded a main effect for congruency, $F(1,41)=32.57, p<$ $.001, d_{\mathrm{z}}=.88$, indicating that participants committed more errors in incongruent trials $(M=8.18 \%, S D=5.31)$ as compared to congruent trials $(M=3.69 \%, S D=2.90)$. Neither the main effect for priming, nor the interaction between congruency and priming were significant, $F$ 's $(1,41)<1.18$, p's $>.284$.

Second, in order to test whether there were any order effects, we ran two (one for latencies and one for error rates) additional 2 (Block order: control first vs. money first) $\times 2$ (Congruency: congruent trials vs. incongruent trials) $\times$ 2 (Priming: money vs. control) mixed ANOVAs with block order as between-subject factor, and congruency as well as priming as within-subject factor. When analyzing latencies, neither the interaction between block order and priming, nor the interaction between block order and congruency, nor the three-way interaction, nor the main effect

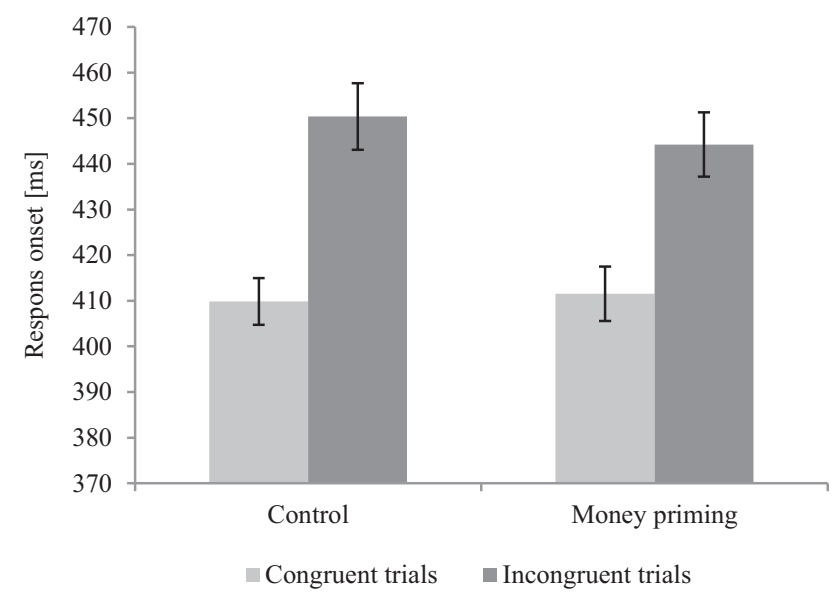

Figure 2. Latencies for congruent and incongruent trials as a function of priming in the pilot study. Error bars represent standard errors of the mean.

for block order was significant, all $F^{\prime} s(1,40)<2.5$, all $p$ 's $>.12$. Likewise, the same effects were not significant when analyzing the error rates, $F^{\prime} \mathrm{s}(1,40)<1.38$, $p$ 's $>$ .24. These results suggest that there was no order effect.

\section{Discussion}

By assessing the imitation-inhibition task (Brass et al, 2001, 2000), the results of the pilot study showed a smaller congruency effect in the money priming condition than in the control condition, thereby providing support for the hypothesis that money priming may indeed increase the focus on the self as compared to others at a basic perceptual-motor level. These results are in line with Vohs et al.'s (2008) theoretical account, arguing that money priming leads individuals to focus more strongly on themselves as compared to others. However, a few critical issues need to be further addressed in order to draw any conclusions.

First, based on previous research suggesting that people reminded of money shift into work mentality (Vohs, 2015), one may argue that money priming increased participants' motivation to be good at the task at hand and that this increase in motivation rather than self-focus drove our effects. Indeed, motivational theories suggest a strong link between motivation and cognitive control (Carver \& Scheier, 2001; Kruglanski et al., 2002; Simon, 1967) - a process that is involved in the imitation-inhibition task as well. Thus, one could argue that increased working mentality may potentially explain the interaction between priming and congruency. However, it is important to note that a person's motivation is based on the activation and representation of specific cognitive and behavioral goals (Kruglanski et al., 2002). In line with this notion, research has shown 
that increased motivation speeds up participants' reaction time in various different tasks (e.g., Douglas \& Parry, 1983; Obitz, Rhodes, \& Creel, 1977; Zink, Pagnoni, Martin-Skurski, Chappelow, \& Berns, 2004). As the goal in the imitation-inhibition task is to respond as fast as possible to the target letters on the screen, the increased working mentality hypothesis would have also predicted a main effect of priming. That is, the more participants are motivated to be good in the task, the faster they should respond to the letters on the screen. As this was not the case in our experiment, we regard the alternative explanation of increased working mentality as rather unlikely.

Second, it might be that attentional processes triggered our effect (Bach \& Tipper, 2007; Cracco, Bardi, et al., 2018; Cracco \& Brass, 2017). That is, money priming may have grasped participants' attention so participants did not focus on the other person's hand anymore. However, we do not regard this alternative explanation as plausible, because participants' task was to respond to the letters on the screen and not to the observed movements. Therefore, if money would have grasped participants' attention, one would also expect slower responses and more errors in the money condition than in the control condition for both congruent and incongruent trials. This, however, was not the case, indicating that the money prime did not grasp more attention than the control prime. Nevertheless, it might still be that money distracted participants' attention from the hand, but not necessarily from the target letter. The random assembly of pixels in our control condition might, thus, not have been the ideal control condition.

Third, our results leave open whether the effects are driven by (a) reduced interference on incongruent trials, (b) reduced facilitation on congruent trials, or (c) both (i.e., increased self-focus affects both congruent and incongruent trials to equal extent). A possible way to investigate this open question would be to add neutral trials, in which the model's hand is not moving, into the design. This would allow computing the interference effect - individuals' tendency to respond slower to incongruent trials than to neutral trials - as well as the facilitation effect - individuals' tendency to respond faster to congruent trials than to neutral trials (cf. Brass et al., 2001, 2000).

Fourth, we did not find any effects of the order of priming conditions. However, this might be due to the small sample in the pilot study, or because there are no order effects.

Fifth, recently, it has been suggested that researchers themselves must provide direct replications of their own effects in order to deal with the unreliability of a single study (Cesario, 2014). Due to the natural fluctuation of $p$-values in significance testing, every study is prone to Type I and Type II errors and might therefore yield unreliable results (Cumming, 2008). In other words, empirical findings might reflect empirical artifacts rather than the underlying truth because the statistical results vary as a function of measurement error and sampling error (see Stanley \& Spence, 2014). This issue is especially important in our case, because the $p$-value of the critical statistical test in the pilot study was with $p=.05$ only approaching significance. The likelihood to obtain a finding that reflects the underlying truth can be increased through larger sample sizes and measures with higher reliability. To minimize measurement error in our pilot study, we already used a reliable trial-by-trial measure instead of a one-trial behavioral measure. However, it is important to note that despite decreasing sampling error in our pilot study, our finding might still be a false-positive. Moreover, although we controlled for color information in the control condition, it might still be that the money condition guided participants' attention away from the model's hand, decreasing the congruency effect. Thus, to cope with these problems and to get further insight into the question whether the effect is driven by interference, facilitation, or both, we ran a highly powered preregistered replication with additional neutral trials in which the model's hand was not moving. In addition, we included order of the priming conditions as a factor into the preregistered analysis to test for possible order effects in a large sample. Finally, a meta-analysis was carried out to cross-validate our observed effects. Specifically, we ran a meta-analysis on all previous, published imitationinhibition experiments that used manipulations related to self-other focus to compare their effect size with the observed effect size in the money condition.

\section{Preregistered Experiment}

Based on the results of the pilot study, we expected smaller congruency effects in the money priming condition as compared to the control condition. With regard to facilitation and interference effects we did not have any clear prediction. In order to test our hypotheses, we conducted a highly powered preregistered experiment. We preregistered the experiment at the Open Science Framework (OSF; https:// osf.io/ncvfs/?view_only=82162828bf194135b8bb63b483ed 2870) after initial acceptance of the registered report on Stage 1 of the review process and before data collection.

\section{Method}

\section{Participants}

In order to estimate the sample size, we conducted a power analysis using $G^{*}$ Power 3.1 (Faul, Erdfelder, Lang, \& Buchner, 2007). The power analysis was set up to detect the smallest effect size of interest, as determined by 
theoretical assumptions and practical limitations (Lakens, 2014). This procedure resulted in an assumed effect size of $d=0.30$, which can be classified following Cohen's (1969) rules of thumb as an effect that is slightly larger than a "small" effect $(d=0.20)$ but substantially smaller than a "medium" effect $(d=0.50)$. Theoretically, this effect size is consistent with a recent meta-analysis showing that the effect of social priming on behavior is around $d=0.30$ (Weingarten et al., 2016). When using $G^{*}$ Power to estimate the sample size needed to detect effect sizes of $d_{\mathrm{z}}=.30$ in a dependent samples $t$-test (two-tailed) with a power of $1-\beta=.95$ and an alpha error probability of $\alpha=$ .05 , the resulting sample size is $N=147$ participants.

In line with this power analysis, 147 subjects ( 71 female, 75 male, 1 diverse) with a mean age of $24.13(S D=6.84)$ participated in the experiment. All subjects were recruited at the University of Cologne (Germany) and received $5 €$ in return for their participation. One hundred and thirtyseven participants were right-handed and 10 participants were left-handed.

\section{Stimuli and Apparatus}

The imitation-inhibition task (Brass et al., 2001, 2000) was programmed using E-Prime software (Schneider, Eschman, \& Zuccolotto, 2002) and was run on custom-build computers with $24^{\prime \prime}$ Benq monitors. Although the preregistered study was very similar to the pilot study, it differed in some details. First, we used a different device to detect participants' responses. As the participant number was rather high and our laboratory does not have more than two custombuilt response boxes with light sensors, we used Apple keyboards to detect participants' responses. Specifically, at the beginning of each trial, participants were asked to press down the "G" key with their right index finger and the " $\mathrm{H}$ " key with their right middle finger. In line with the pilot study, participants were instructed to lift their index finger in response to the letter $A$ and their middle finger in response to the letter $\mathrm{B}$. The use of keyboards to measure automatic-imitation has been validated in many different studies (e.g., Aicken, Wilson, Williams, \& Mon-Williams, 2007; Boyer, Longo, \& Bertenthal, 2012; Butler, Ward, \& Ramsey, 2015; Santiesteban, Banissy, Catmur, \& Bird, 2015; Sowden, Koehne, Catmur, Dziobek, \& Bird, 2015).

Second, we used different stimuli. That is, instead of a hand that is perceived as clearly female, we used a female hand that looked more gender-neutral. This is supported by a pretest in which 54 participants ( 23 males) with an age ranging from 18 to $66(M=37.04 ; S D=11.32)$ indicated on 7-point rating scales $(1=$ not at all to $7=$ very $m u c h)$ how strongly the attributes "feminine" and "masculine" apply to the hand. A $t$-test for dependent samples indicated that participants did not perceive the hand as more feminine $(M=4.44 ; S D=1.53)$ than masculine $(M=4.07 ; S D=$

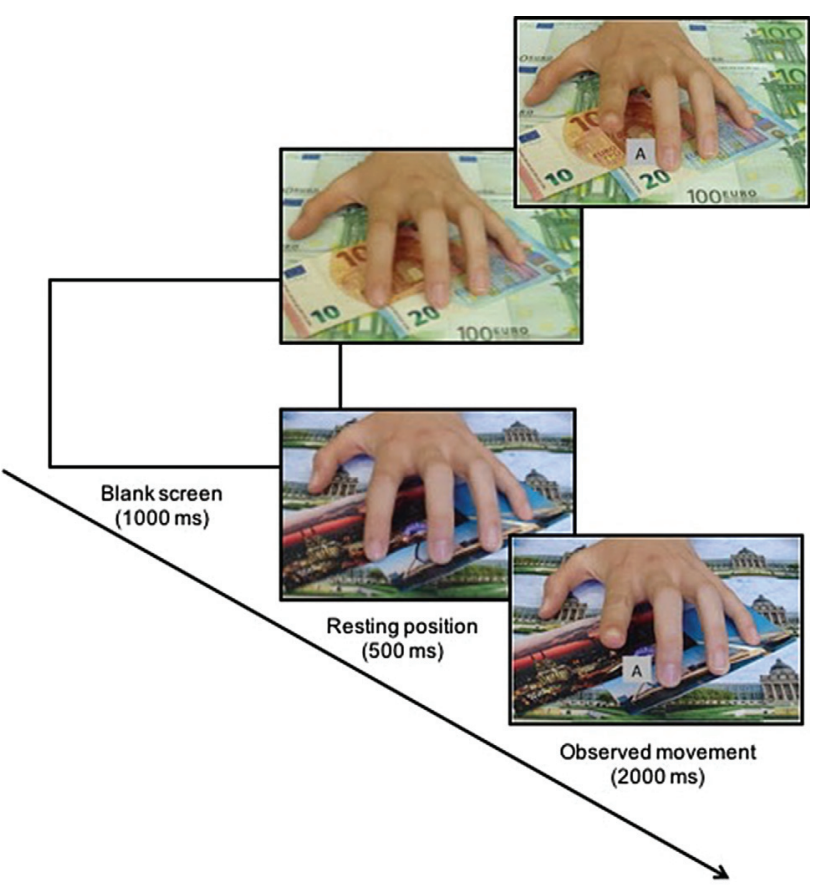

Figure 3. Stimuli and procedure of the preregistered study.

1.65), $t(53)=0.99, p=.33$. Furthermore, although the background in the money condition was similar to the pilot study, we changed the background in the control priming condition. Specifically, the model's hand was placed on a bunch of different postcards. The stimulus materials in both the money priming condition and the control condition were shown in color and were identical in terms of size $(444 \times$ 286 pixels). In each condition the model's hand was always laying on the same background (i.e., same money bills or same postcards). Figure 3 illustrates the stimulus material.

Third, in order to test whether the effects of the pilot study were driven by (a) reduced interference on incongruent trials, (b) reduced facilitation on congruent trials, or (c) both, we added neutral trials into the design, in which the model's hand was not lifting any finger. This allowed us to compute, in addition to the congruency effect, the interference effect - individuals' tendency to respond slower to incongruent trials than to neutral trials - and the facilitation effect - individuals' tendency to respond faster to congruent trials than to neutral trials (cf. Brass et al., 2001, 2000).

\section{Procedure}

The procedure was similar to the pilot study. First, participants signed an informed consent. Second, participants ran through the imitation-inhibition task. There was no actual cover story. Instead, participants read specific instructions about the imitation-inhibition task. That is, they were instructed to respond as fast and as accurate as possible to the letters " $\mathrm{A}$ " and " $\mathrm{B}$ " on the screen ( $\mathrm{A}=$ lift index finger, $\mathrm{B}=$ lift middle finger). Afterward, participants 
performed two blocks of the imitation-inhibition task. In one block the observed hand was lying on money bills and in one block on postcards. Within each block, the background (i.e., money bills or postcards) was always the same. In order to get familiar with the task, participants ran through eight practice trials before each experimental block. Between the two experimental blocks, participants were allowed to take a small break. The order of the experimental blocks was counterbalanced across participants and each block consisted of 120 trials (i.e., 40 congruent trials, 40 neutral trials, and 40 incongruent trials). The total amount of experimental trials was 240 . Finally, participants indicated basic demographic data - namely gender, age, and handedness - were fully debriefed by the experimenter and were then dismissed.

\section{Imitation-Inhibition Task}

Participants had to lift their right index or middle finger in response to a letter $(\mathrm{A}=$ lift index finger, $\mathrm{B}=$ lift middle finger) on the computer screen while watching a congruent finger movement, incongruent finger movement, or no movement. All trials were presented randomly. Each trial started with the appearance of a picture showing a gender-neutral hand mirroring participants' right hand in resting position for $500 \mathrm{~ms}$. Afterward, a second picture of the model lifting either the index finger, lifting the middle finger, or not lifting any finger was presented for $2000 \mathrm{~ms}$ or until participants responded. Simultaneously, the imperative cue (i.e., letter A or B) was displayed between the model's index and middle finger. Between trials, the screen was blank for $1000 \mathrm{~ms}$.

\section{Data Preparation}

Exclusion of participants and preparation of the data was conducted in accordance with the pilot study and the preregistration. Responses were detected for all participants and none of the participants committed errors in more than one third of the trials. Moreover, none of the participants had an average response speed that deviated more than three $S D$ s from the sample mean. In line with the pilot study and with past research on the same task (e.g., Catmur \& Heyes, 2011; Cracco et al., 2015), we excluded trials with RT faster than $100 \mathrm{~ms}(0.4 \%)$ and RT slower than 1000 ms (1.0\%). For the RT analyses, erroneous trials (5.2\%) were excluded as well.

\section{Reliability}

In order to test the reliability of the imitation-inhibition task we assessed split-half reliability. First, we computed for even trials and for odd trials the congruency effect (difference between congruent and incongruent trials), the interference effect (difference between neutral and incongruent trials), and the facilitation effect (difference between congruent and neutral trials), and then computed the Spearman-Brown coefficient. The results yielded $\rho^{*}=.80$ for the congruency effect, $\rho^{*}=.62$ for the interference effect, and $\rho^{*}=.55$ for the facilitation effect.

\section{Results}

\section{Preregistered Confirmatory Analyses}

\section{Latencies}

To test our predictions, we conducted a 2 (Block order: control first vs. money first) $\times 2$ (Congruency: congruent trials vs. incongruent trials) $\times 2$ (Priming: money vs. control) mixed ANOVA with block order as between-subject factor, and congruency as well as priming as within-subject factor (for an overview of means and standard deviations see Table 1). The results yielded a main effect for congruency, $F(1,145)=483.97, p<.001, d_{\mathrm{z}}=1.83$, indicating that participants responded faster (in ms) on congruent trials $(M=467.80 ; S D=49.18)$ than on incongruent trials $(M=529.54 ; S D=61.14)$. The main effects for priming, $F(1,145)=0.29, p=.593$, and block order, $F(1,145)=$ $0.92, p=.340$, were not significant.

More important for our predictions, however, was the significant interaction between congruency and priming, $F(1,145)=5.13, p=.025, d_{\mathrm{z}}=.19$, indicating smaller congruency effects in the money priming condition $(\Delta=59.35$; $S D=38.03)$ than in the control condition $(\Delta=64.13$; $S D=39.32)$. The results are depicted in Figure 4 .

Block order did not interact with congruency, $F(1,145)=$ $0.003, p=.957$. However, the two-way interaction between block order and priming, $F(1,145)=11.72, p=.001, \eta_{\mathrm{p}}{ }^{2}=.08$ as well as the three-way interaction between block order, congruency and priming was significant, $F(1,145)=19.76$, $p<.001, \eta_{\mathrm{p}}{ }^{2}=.12$. Non-preregistered post-hoc analyses shedding light onto this interaction indicated that when the control block was assessed first, the congruency effect was larger in the control condition $(\Delta=72.11 ; S D=43.58)$ than in the money priming condition $(\Delta=51.72 ; S D=$ 35.83), $F(1,61)=17.45, p<.001, d_{\mathrm{z}}=.53$. In contrast, there was a trend into the opposite direction $\left(\Delta_{\text {control }}=58.30\right.$; $\left.S D_{\text {control }}=35.03 ; \Delta_{\text {money }}=64.92 ; S D_{\text {money }}=38.83\right)$ when the money block was assessed first, $F(1,84)=3.07, p=$ $.083, d_{\mathrm{z}}=.19$.

\section{Preregistered Explorative Analyses}

\section{Error Rates}

In a first preregistered explorative analysis we ran the same analysis for error rates that we conducted for latencies. A 2 (Block order: control first vs. money first) $\times 2$ (Congruency: congruent trials vs. incongruent trials) $\times 2$ 
Table 1. Preregistered experiment: Means and standard deviations of the imitation-inhibition task within the control and the money priming condition

\begin{tabular}{lrrrrr}
\hline & \multicolumn{2}{c}{ Control } & & \multicolumn{2}{c}{ Money priming } \\
\cline { 2 - 3 } & $M$ & $S D$ & & $M$ & $S D$ \\
\hline Congruent trials & 465.10 & 48.46 & & 470.49 & 55.50 \\
Neutral trials & 489.16 & 54.81 & & 493.85 & 55.48 \\
Incongruent trials & 529.23 & 63.39 & & 529.84 & 66.70 \\
Congruency effect & 64.13 & 39.32 & & 59.35 & 38.03 \\
Facilitation effect & 24.06 & 26.69 & & 23.36 & 26.87 \\
Interference effect & 40.07 & 32.60 & & 35.99 & 35.14 \\
\hline
\end{tabular}

Note. $M=$ Mean, $S D=$ Standard Deviation.

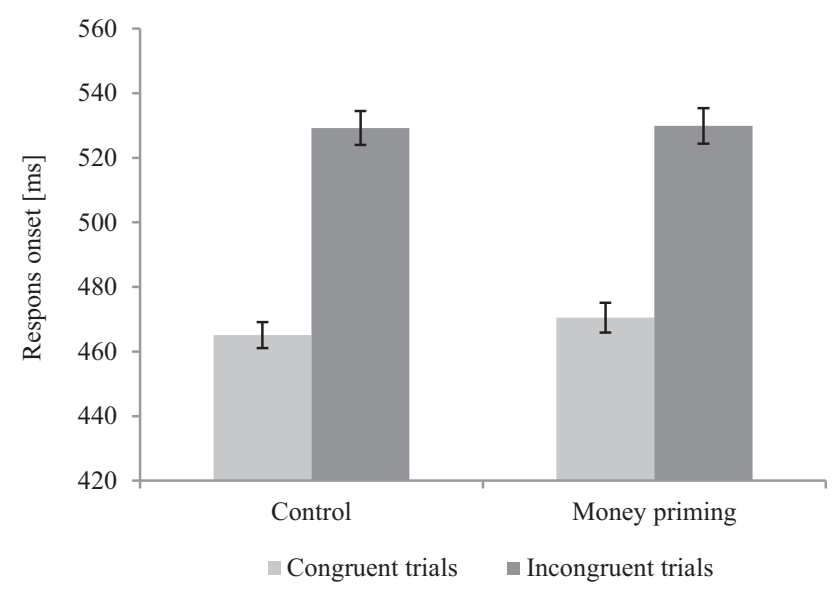

Figure 4. Latencies for congruent and incongruent trials as a function of priming in the preregistered experiment. Error bars represent standard errors of the mean.

(Priming: money vs. control) mixed ANOVA with block order as between-subject factor, and congruency as well as priming as within-subject factor yielded a main effect for congruency $F(1,145)=158.49, p<.001, d_{\mathrm{z}}=1.05$, indicating that participants made more errors on incongruent trials $(M=8.73 \% ; S D=6.76)$ than on congruent trials $(M=$ $3.05 \% ; S D=4.16)$. Neither the main effect of block order, nor the main effect of priming, nor the interaction between congruency and block order, nor the interaction between priming and block order was significant, $F$ 's $(1,145)<0.57$, p's $>.45$.

More important for our hypothesis was the interaction between congruency and priming, which approached significance, $F(1,145)=3.64, p=.058, d_{\mathrm{z}}=.16$. This indicates that the congruency effect was tendentially smaller in the money priming condition $(\Delta=5.24 ; S D=6.44)$ as compared to the control condition $(\Delta=6.12 ; S D=6.86)$.

Similar to the analyses of the latencies, the three-way interaction between block order, congruency and priming was significant, $F(1,145)=10.50, p=.001, \eta_{\mathrm{p}}{ }^{2}=.07$. Based on non-preregistered post-hoc analyses, this indicates that the two-way interaction between congruency and priming was significant when the control block was assessed first, $F(1,61)=13.10, p=.001, d_{\mathrm{z}}=.46$, but not when the money block was assessed first, $F(1,84)=0.97, p=.328$.

\section{Cross-Validation With Previous Findings}

In order to cross-validate our findings, we descriptively compared the detected effect size on the latencies in the preregistered study with the average effect size of all previously published experiments (i.e., before in principle acceptance of this paper - i.e., before March 29, 2018) that used manipulations related to self-other focus and the imitationinhibition task as dependent variable. In a first step we ran a meta-analysis. Specifically, we included all published papers (for an overview, see Table 1 in Electronic Supplemental Material, ESM 1) in which the imitation-inhibition task was performed by an adult sample after self-focus (or related) priming, control priming, or both (Cook \& Bird, 2011, 2012; Hogeveen \& Obhi, 2011; Leighton et al., 2010; Spengler et al., 2010; Wang \& Hamilton, 2013, 2015).

Since research has shown that social priming effects may reverse when the primes are presented in a third-person perspective (Wang \& Hamilton, 2013, 2015), we did not include effect sizes obtained with third-person primes. This procedure resulted in a sample of 10 experiments from seven studies that could be included in the meta-analysis. Nine of these experiments used self-focus primes $(N=$ $155)$ and five experiments used control primes $(N=101)$.

For three experiments, we were able to extract all relevant information from the paper (Cook \& Bird, 2011, 2012; Hogeveen \& Obhi, 2011). For another three experiments, we could calculate effect sizes on the basis of the raw data (Wang \& Hamilton, 2013). Finally, the effect sizes of the final four experiments (Leighton et al., 2010; Spengler et al., 2010; Wang \& Hamilton, 2015) were extracted from the graphs using a computerized measurement tool. Because four experiments provided multiple effect sizes from the same sample of participants (e.g., within-subject manipulation), we used robust variance estimation with correlated effects weights and a small sample correction to account for the fact that these effect sizes were correlated (Tanner-Smith, Tipton, \& Polanin, 2016). A mixed effects model with a moderator for condition revealed a significant difference between the congruency effect after self-focus priming $\left(d_{\mathrm{z}}=0.85\right)$ compared with control priming $\left(d_{\mathrm{z}}=1.43\right), t(5.37)=3.75, p=.012$ (Figure 5). However, one could argue that priming in the Wang and Hamilton studies was less strongly related to self-other focus than the primes in the other studies. Thus, we also repeated the meta-analysis without these studies. This revealed that effect size of the difference between the self-focus congruency effect $\left(d_{z}=0.96\right)$ and control congruency effect $\left(d_{z}=1.73\right)$ was even larger without than with these studies, $t(2.86)=7.72, p=.005$. 


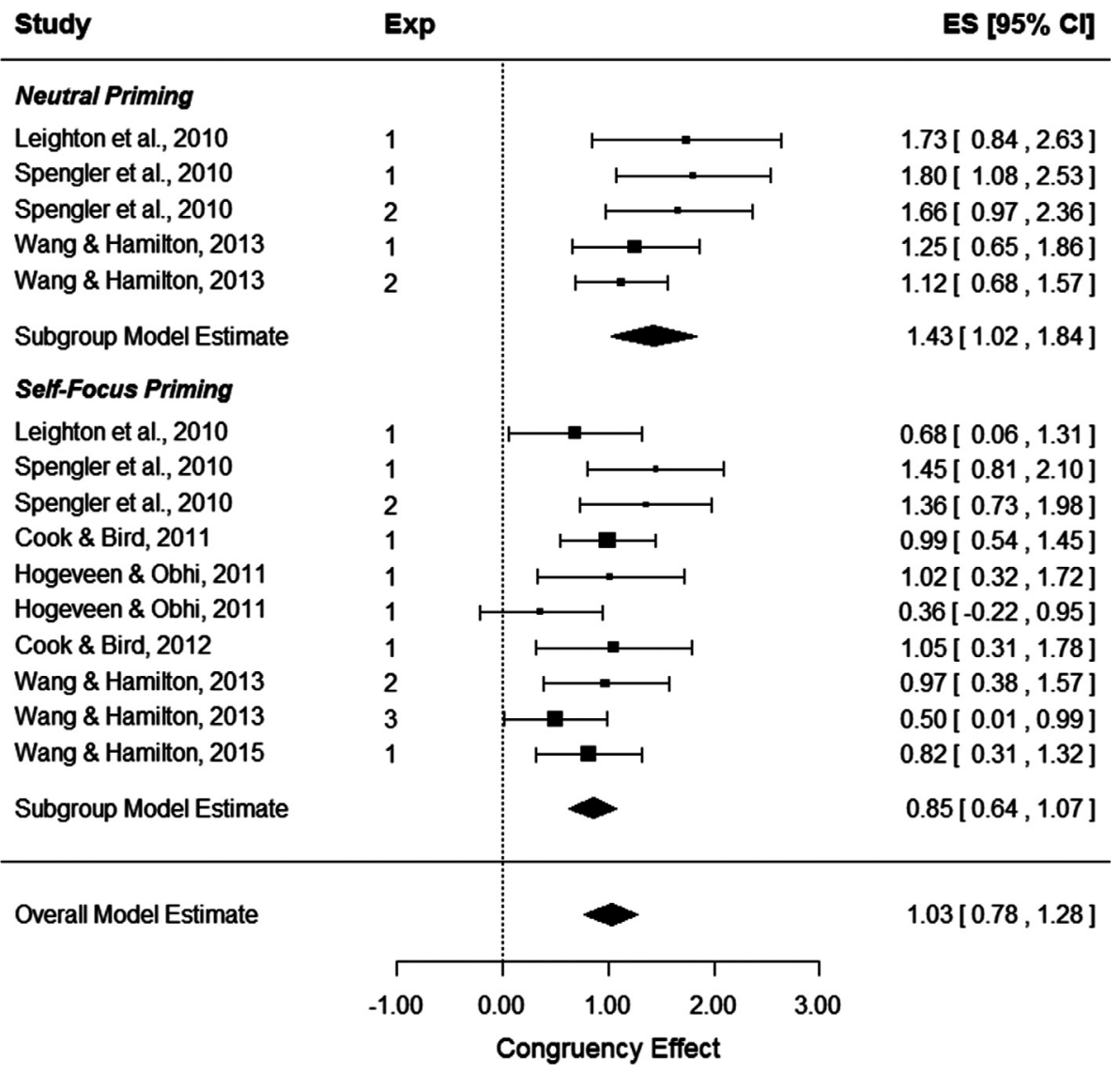

Figure 5. Forest plot for the relation between the reaction time based congruency effect in the imitation-inhibition task (Brass et al., 2001, 2000) and self-other focus. Effect sizes are $d_{z}$.

In the next step, we computed for both, the meta-analysis and the preregistered experiment, the difference in $d_{\mathrm{z}}$ between the experimental condition and the control condition. This revealed $d_{z}=.58$ for the meta-analytic effect and $d_{\mathrm{z}}=.19$ for the preregistered experiment suggesting that money priming is less effective in inducing self-focus than conventional methods used in previous research.

\section{Interference and Facilitation}

In order to investigate the role of interference and facilitation in the preregistered experiment, we first computed, separately for the money and control priming condition, the interference effect by subtracting the mean value on neutral trials from the mean value on incongruent trials, and the facilitation effect by subtracting the mean value within congruent trials from the mean value within neutral trials. Even though we had no clear hypothesis on whether the expected interaction effect between priming and congruency should be due to interference, facilitation, or both, this exploratory analysis aimed at shedding more light onto the underlying processes of the observed effect. To this end, we ran for latencies as well as for error rates a 3 (Congruency: congruent trials vs. neutral trials vs. incongruent trials) $\times 2$ (Priming: money vs. control) repeated measure ANOVA.

The ANOVA for latencies yielded a main effect of congruency, $F(2,145)=382.22, p<.001, d_{\mathrm{z}}=1.62$, indicating that participants responded faster on congruent trials $(M$ $=467.80 ; S D=49.18)$ than on neutral trials $(M=491.51$; $S D=52.05)$, and faster on neutral trials than on incongruent trials $(M=529.54 ; S D=61.14), t$ 's $(146)>13.66$, $p$ 's < $.001, d_{\mathrm{z}}$ 's $>1.12$. Neither the main effect of priming, nor the interaction between congruency and priming was significant, $F$ 's $<1.88$, $p$ 's $>$.17. Further preregistered exploratory $t$-tests detected no significant difference between the money priming condition and the control condition in terms of the interference or facilitation effect, $t^{\prime} s(146)<1.14, p$ 's $>.25$.

The same ANOVA for error rates found a main effect for congruency, $F(2,145)=129.43, p<.001, d_{\mathrm{z}}=.94$, indicating that participants made fewer errors on congruent trials $(M=$ $3.05 \% ; S D=4.16)$ than on neutral trials $(M=4.04 \%$; $S D=$ $4.11)$, and fewer errors on neutral trials than on incongruent trials $(M=8.73 \%$; $S D=6.76), t^{\prime} s(146)>4.05, p$ 's $<.001$, $d_{\mathrm{z}}$ 's $>$.33. Neither the main effect of priming, nor the 
interaction between congruency and priming was significant, $F$ 's $<2.30, p$ 's $>.12$. Further preregistered exploratory $t$-tests found a stronger facilitation effect in the control condition $(\Delta=1.58 ; S D=4.54)$ as compared to the money priming condition $(\Delta=0.41 ; S D=4.08), t(146)=2.28, p=$ $.024, d_{\mathrm{z}}=.19$. There was no difference in terms of interference between the money priming condition and the control condition, $t(146)=0.47, p=.637$.

\section{Non-Preregistered Explorative Bayes Analyses}

The crucial preregistered hypothesis test (i.e., interaction between priming and congruency) replicated the findings obtained in the pilot study. To assert the likelihood of our data under H1 (i.e., money primes lead to smaller congruency effects than control primes) relative to HO, we calculated Bayes factor using Dienes (cf. Dienes, 2014; Dienes, Coulton, \& Heather, 2018) online calculator (http://www. lifesci.sussex.ac.uk/home/Zoltan_Dienes/inference/bayes_ factor.swf). Specifically, we tested how likely $\mathrm{H} 1$ is given the effect obtained in the pilot study. This required entering the mean difference between the congruency effect of the control condition and the money condition $(M=4.78 ; S E=$ 2.12). We tested with a halfnormal distribution centered on 0 and with a $S D$ of 7.89 , which corresponds to the mean difference obtained in the pilot study. This approach yielded a $\mathrm{BF}=5.48$ indicating that the data is 5.48 times more likely to have occurred under H1 than under HO. A Bayes factor between 3 and 10 is conventionally considered to be moderate evidence (Jeffreys, 1961).

\section{General Discussion}

According to the self-sufficiency hypothesis (Vohs et al., 2006), people who are reminded of money evaluate options and actions in terms of personal inputs and outputs. Consequently, when primed with money, people focus more strongly on self-related needs and less on the needs of others (Reutner \& Wänke, 2013; Schuler \& Wänke, 2016; Vohs et al., 2008). However, several studies (Klein et al., 2014; Rohrer et al., 2015; Schuler \& Wänke, 2016) could not replicate some of the findings reported in the money priming literature.

A reason for these inconsistent findings may lay in the common use of explicit self-report measures. These measures are prone to demand effects and tend to rely on one or just a few items, leading to low reliability. Therefore, we tested the core assumption underlying the self-sufficiency hypothesis (i.e., money primes lead to an increased focus on the self; Vohs et al., 2006), with the imitation-inhibition task (Brass et al., 2001, 2000) - a reliable and unobtrusive trial-by-trial task, which is sensitive to self-other focus. Moreover, to strengthen the effect of the manipulation, in contrast to previous research, we presented the money primes on each trial.

A pilot study found a smaller congruency effect, and therefore a stronger focus on the self, in the money priming condition than in the control condition. A preregistered, high-powered experiment replicated this effect, suggesting that money primes may indeed increase a focus on the self. However, due to several limitations, these results need to be interpreted with caution and call for further investigations.

First, the results in the preregistered experiment are strongly influenced by the order of the priming conditions. Significant effects in the expected direction were detected only when the control condition was assessed first, but not when the money priming condition was assessed first. That is, when the control block was assessed first, the congruency effect was larger in the control condition as compared to the money condition. In contrast, when the money block was assessed first, there was a nonsignificant tendency that the congruency effect was larger in the money priming condition as compared to the control condition. There are different potential explanations for this effect. First, it is reasonable to assume that we faced spillover effects. That is, when the money block was assessed first, the money primes induced a focus on the self which persisted in the control block working against our predictions. Second, it might be that the money primes induced a focus on the self, due to contrastive, or comparative processes (e.g., Mussweiler, 2003). That is, only when money primes can be contrasted or compared to another prime (i.e., the control condition in our experiments), money primes may induce a focus on the self. Third, one may argue that over time, participants got better at the task (cf. Cracco, Bardi, et al., 2018), which could have reduced the congruency effect, and therefore potentially also the effect of money primes, in the second block. However, we regard this explanation as rather unlikely, because only the three-way interaction between block order, congruency, and priming, but not the two-way interaction between block order and congruency was significant.

Second, another limitation concerns the way we analyzed the data. In this respect, it is important to note that the Critical money priming $\times$ Congruency interaction in the preregistered experiment is significant only when we apply the preregistered analysis. For example, when removing block order from the analysis in an exploratory analysis, the Money priming $\times$ Congruency interaction becomes nonsignificant $\left(p_{\text {latencies }}=.137\right.$; $p_{\text {error rate }}=.172$ ). This indicates that the results might not be very robust and strongly rely on the way the data are analyzed. 
Third, even if one would regard our findings as support for the self-sufficiency hypothesis (Vohs et al., 2006), we need to acknowledge that the found effects are with $d_{\mathrm{z}}=$ .19 rather small. Indeed, a cross validation with previous experiments indicates that priming participants with money produces rather weak self-focus effects as compared to other manipulations, such as scrambled sentences tasks (Cook \& Bird, 2011; Leighton et al., 2010; Wang \& Hamilton, 2013, 2015), word priming tasks (Hogeveen \& Obhi, 2011), or mirror manipulations and self-referential tasks (Spengler et al., 2010). This is in line with Caruso et al. (2017) who found in three high-powered studies only weak evidence for the idea that money primes increase a focus on the self.

Fourth, our results remain inconclusive in regard to the question of whether money priming increases a focus on the self due to facilitation or interference. That is, when computing interference and facilitation effects, we did not find a significant difference between the money priming and the control condition in terms of facilitation and interference. Given that facilitation and interference effects measure a part of the congruency effect and are therefore smaller (cf. Cracco, Bardi, et al., 2018) and given that the effect of money priming is rather weak, future research may increase the number of participants to detect small effect sizes.

Fifth, one may argue that the found differences between the money priming and the control condition are due to attentional processes. That is, it could be that the money primes grasped participants' attention so that participants did not focus on the other person's hand anymore. However, we do not regard this alternative explanation as plausible for different reasons. On the one hand, participants' task was to respond to the letters on the screen and not to the observed movements. Therefore, if money would have grasped participants' attention, one would have expected that participants respond, on average, slower and with more errors in the money condition than in the control condition. However, in both experiments, this was not the case. On the other hand, in the preregistered experiment, we made sure that the background in the control condition was similarly attention-grabbing as the background in the money priming condition. Nevertheless, it could in principle be that when using other stimuli, the magnitude of the effect will change. Future research may, thus, use a variety of different stimuli to cross validate our findings and to test its generalizability.

\section{Theoretical Implications}

Although we found only weak evidence for the idea that money priming increases a focus on the self, our results nevertheless have important theoretical implications. First, our results may explain why previous research had difficulties to replicate earlier work in the field of money priming. In our research, we used high-powered within-subject designs, integrated the primes with the stimuli, and assessed a large number of participants. Despite our effort to maximize the chance of finding support for the selfsufficiency hypothesis, we detected in line with previous research (Caruso et al., 2017) only small effects. And these small effects could only be detected when specific preconditions (e.g., manipulation of block order) were met. Thus, it is not surprising that when using less powerful betweensubject designs, applying less reliable measures, and implementing weaker manipulations, it is rather difficult to find effects of money primes.

Second, our results also have implications for research on imitation by contributing to the current discussion on the role of social top-down influences (that are not necessarily related to self-other focus). Recently, researchers have suggested that imitation may not be as strongly modulated by certain social top-down influences as previously assumed (e.g., Cracco, Bardi, et al., 2018; Cracco \& Brass, 2019; Genschow et al., 2017; Ramsey, 2018). In contrast, our carried out meta-analysis indicates that automatic imitation can be modulated if the moderators are related to self-other focus. Moreover, the data of our experiments suggest that the imitation-inhibition task can be influenced by (social) top-down modulations when assessing large samples. This is consistent with the results of a recent study that found similar effects of social priming on the imitation-inhibition task in two high-powered experiments (Cracco, Genschow, et al., 2018).

\section{Electronic Supplementary Material}

The electronic supplementary material is available with the online version of the article at https://doi.org/ 10.1027/1618-3169/a000466

ESM 1. Additional table, additional analyses, and references

\section{References}

Aicken, M. D., Wilson, A. D., Williams, J. H., \& Mon-Williams, M. (2007). Methodological issues in measures of imitative reaction times. Brain and Cognition, 63, 304-308. https://doi.org/ 10.1016/j.bandc.2006.09.005

Bach, P., \& Tipper, S. P. (2007). Implicit action encoding influences personal-trait judgments. Cognition, 102, 151-178. https://doi. org/10.1016/j.cognition.2005.11.003

Boucher, H. C., \& Kofos, M. N. (2012). The idea of money counteracts ego depletion effects. Journal of Experimental 
Social Psychology, 48, 804-810. https://doi.org/10.1016/ j.jesp.2012.02.003

Boyer, T. W., Longo, M. R., \& Bertenthal, B. I. (2012). Is automatic imitation a specialized form of stimulus-response compatibility? Dissociating imitative and spatial compatibilities. Acta Psychologica, 139, 440-448. https://doi.org/10.1016/j. actpsy.2012.01.003

Brass, M., Bekkering, H., \& Prinz, W. (2001). Movement observation affects movement execution in a simple response task. Acta Psychologica, 106, 3-22.

Brass, M., Bekkering, H., Wohlschläger, A., \& Prinz, W. (2000). Compatibility between observed and executed finger movements: Comparing symbolic, spatial, and imitative cues. Brain and Cognition, 44, 124-143. https://doi.org/10.1006/ brcg.2000.1225

Brass, M., Ruby, P., \& Spengler, S. (2009). Inhibition of imitative behaviour and social cognition. Philosophical Transactions of the Royal Society B: Biological Sciences, 364, 2359-2367. https://doi.org/10.1098/rstb.2009.0066

Butler, E. E., Ward, R., \& Ramsey, R. (2015). Investigating the relationship between stable personality characteristics and automatic imitation. PLoS One, 10, e0129651. https://doi.org/ 10.1371/journal.pone.0129651

Caruso, E. M., Shapira, O., \& Landy, J. F. (2017). Show me the money: A systematic exploration of manipulations, moderators, and mechanisms of priming effects. Psychological Science, 28, 1148-1159. https://doi.org/10.1177/0956797617706161

Carver, C. S., \& Scheier, M. F. (2001). On the self-regulation of behavior. Cambridge, UK: University Press.

Catmur, C., \& Heyes, C. (2011). Time course analyses confirm independence of imitative and spatial compatibility. Journal of Experimental Psychology: Human Perception and Performance, 37, 409-421. https://doi.org/10.1037/a0019325

Cesario, J. (2014). Priming, replication, and the hardest science. Perspectives on Psychological Science, 9, 40-48. https://doi. org/10.1177/1745691613513470

Cohen, J. (1969). Statistical power analysis for the behavioural sciences. New York, NY: Academic Press.

Cook, J., \& Bird, G. (2011). Social attitudes differentially modulate imitation in adolescents and adults. Experimental Brain Research, 211, 601-612. https://doi.org/10.1007/s00221-011-2584-4

Cook, J. L., \& Bird, G. (2012). Atypical social modulation of imitation in autism spectrum conditions. Journal of Autism and Developmental Disorders, 42, 1045-1051. https://doi.org/ 10.1007/s10803-011-1341-7

Cracco, E., Bardi, L., Desmet, C., Genschow, O., Rigoni, D., De Coster, L., ... Brass, M. (2018). Automatic imitation: A metaanalysis. Psychological Bulletin, 5, 453-500. https://doi.org/ 10.1037/bul0000143

Cracco, E., \& Brass, M. (2017). Automatic imitation of multiple agents: Simultaneous or random representation? Journal of Experimental Psychology, 44, 729-740. https://doi.org/ $10.1037 / x h p 0000489$

Cracco, E., \& Brass, M. (2019). Reaction time indices of automatic imitation measure imitative response tendencies. Consciousness and Cognition, 68, 115-118. https://doi.org/10.1016/ j.concog.2019.01.001

Cracco, E., Clauwaert, A., Van den Broeck, Y., Van Damme, S., \& Brass, M. (2019). Motor simulation is disturbed when experiencing pain. Pain, 160, 2743-2750. https://doi.org/10.1097/ j.pain. 0000000000001665

Cracco, E., De Coster, L., Andres, M., \& Brass, M. (2015). Motor simulation beyond the dyad: Automatic imitation of multiple actors. Journal of Experimental Psychology: Human Perception and Performance, 41, 1488-1501. Empathy results retrieved from https://osf.io/eas $1484 \mathrm{~m}$
Cracco, E., Genschow, O., Radkova, I., \& Brass, M. (2018). Automatic imitation of pro- and antisocial gestures: Is implicit social behavior censored? Cognition, 170, 179-189.

Cumming, G. (2008). Replication and $p$ intervals: $p$ values predict the future only vaguely, but confidence intervals do much better. Perspectives on Psychological Science, 3, 286-300. https://doi.org/10.1111/j.1745-6924.2008.00079.x

Dienes, Z. (2014). Using Bayes to get the most out of nonsignificant results. Frontiers in Psychology, 5, 781.

Dienes, Z., Coulton, S., \& Heather, N. (2018). Using Bayes factors to evaluate evidence for no effect: Examples from the SIPS project. Addiction, 113, 240-246. https://doi.org/10.1111/ add.14002

Douglas, V. I., \& Parry, P. A. (1983). Effects of reward on delayed reaction time task performance of hyperactive children. Journal of Abnormal Child Psychology, 11, 313-326.

Epstein, S. (1980). The stability of behavior: II. Implications for psychological research. American Psychologist, 35, 790806.

Faul, F., Erdfelder, E., Lang, A.-G., \& Buchner, A. (2007). G* Power 3: A flexible statistical power analysis program for the social, behavioral, and biomedical sciences. Behavior Research Methods, 39, 175-191. https://doi.org/10.3758/bf03193146

Fiske, A. P. (1991). Structures of social life: The four elementary forms of human relations: Communal sharing, authority ranking, equality matching, market pricing. New York, NY: Free Press.

Gaôsiorowska, A., \& Heõõlka, A. (2012). Psychological consequences of money and money attitudes in dictator game. Polish Psychological Bulletin, 43, 20-26. https://doi.org/10.2478/ v10059-012-0003-8

Gasiorowska, A., Zaleskiewicz, T., \& Wygrab, S. (2012). Would you do something for me? The effects of money activation on social preferences and social behavior in young children. Journal of Economic Psychology, 33, 603-608.

Genschow, O., \& Florack, A. (2014). Attention on the source of influence reverses the impact of cross-contextual imitation. Journal of Experimental Psychology: Human Perception and Performance, 40, 904-907. https://doi.org/10.1037/a0035430

Genschow, O., Florack, A., \& Wänke, M. (2013). The power of movement: Evidence for context-independent movement imitation. Journal of Experimental Psychology: General, 142, 763773. https://doi.org/10.1037/a0029795

Genschow, O., \& Schindler, S. (2016). The influence of group membership on cross-contextual imitation. Psychonomic Bulletin \& Review, 23, 1257-1265. https://doi.org/10.3758/ s13423-015-0983-4

Genschow, O., van Den Bossche, S., Cracco, E., Bardi, L., Rigoni, D. \& Brass, M. (2017). Mimicry and automatic imitation are not correlated. PLoS One, 12, e0183784. https://doi.org/10.1371/ journal.pone.0183784

Gino, F., \& Mogilner, C. (2014). Time, money, and morality. Psychological Science, 25, 414-421. https://doi.org/10.1177/ 0956797613506438

Greenwald, A. G. (1970). Sensory feedback mechanisms in performance control: With special reference to the ideo-motor mechanism. Psychological Review, 77, 73-99.

Guéguen, N., \& Jacob, C. (2013). Behavioral consequences of money: When the automated teller machine reduces helping behavior. The Journal of Socio-Economics, 47, 103-104. https://doi.org/10.1016/j.socec.2013.09.004

Hermans, D., De Houwer, J., \& Eelen, P. (2001). A time course analysis of the affective priming effect. Cognition \& Emotion, 15, 143-165. https://doi.org/10.1080/0269993004200033

Hermans, D., Spruyt, A., \& Eelen, P. (2003). Automatic affective priming of recently acquired stimulus valence: Priming at SOA 300 but not at SOA 1000. Cognition \& Emotion, 17, 83-99. 
Hogeveen, J., \& Obhi, S. S. (2011). Altogether now: Activating interdependent self-construal induces hypermotor resonance. Cognitive Neuroscience, 2, 74-82. https://doi.org/10.1080/ 17588928.2010 .533164

Jeffreys, H. (1961). Theory of probability. Oxford, UK: Clarendon Press.

Klein, R. A., Ratliff, K. A., Vianello, M., Adams, R. B. Jr, Bahník, Š., Bernstein, M. J., ... Nosek, B. A. (2014). Investigating variation in replicability: A "many labs" replication project. Social Psychology, 45, 142-152. https://doi.org/10.1027/1864-9335/ a000178

Kouchaki, M., Smith-Crowe, K., Brief, A. P., \& Sousa, C. (2013). Seeing green: Mere exposure to money triggers a business decision frame and unethical outcomes. Organizational Behavior and Human Decision Processes, 121, 53-61. https://doi.org/ 10.1016/j.obhdp.2012.12.002

Kruglanski, A. W., Shah, J. Y., Fishbach, A., Friedman, R., Chun, W. Y., \& Sleeth-Keppler, D. (2002). A theory of goal systems. In M. P. Zanna (Ed.), Advances in experimental social psychology (Vol. 34, pp. 331-378). San Diego, CA: Academic Press.

LaFrance, M. (1982). Posture mirroring and rapport. In M. Davis (Ed.), Interaction rhythms: Periodicity in communicative behavior (pp. 279-298). New York, NY: Human Sciences Press.

Lakens, D. (2014). Performing high-powered studies efficiently with sequential analyses. European Journal of Social Psychology, 44, 701-710. https://doi.org/10.1002/ejsp.2023

Lamm, C., Bukowski, H., \& Silani, G. (2016). From shared to distinct self-other representations in empathy: Evidence from neurotypical function and socio-cognitive disorders. Philosophical Transactions of the Royal Society B, 371, 20150083.

Leighton, J., Bird, G., Orsini, C., \& Heyes, C. (2010). Social attitudes modulate automatic imitation. Journal of Experimental Social Psychology, 46, 905-910. https://doi.org/10.1016/ j.jesp.2010.07.001

Lodder, P., Ong, H. H., Grasman, R. P., \& Wicherts, J. (2019). A comprehensive meta-analysis of money priming. Journal of Experimental Psychology: General, 148, 688-712.

Mok, A., \& De Cremer, D. (2018). Too tired to focus on others? Reminders of money promote considerate responses in the face of depletion. Journal of Business and Psychology, 33, 405421.

Mussweiler, T. (2003). Comparison processes in social judgment: Mechanisms and consequences. Psychological Review, 110, 472-488. https://doi.org/10.1037/0033-295X.110.3.472

Obitz, F. W., Rhodes, L. E., \& Creel, D. (1977). Effect of alcohol and monetary reward on visually evoked potentials and reaction time. Journal of Studies on Alcohol, 38, 2057-2064.

Pashler, H., Rohrer, D., Abramson, I., Wolfson, T., \& Harris, C. R. (2016). A social priming data set with troubling oddities. Basic and Applied Social Psychology, 38, 3-18. https://doi.org/ 10.1080/01973533.2015.1124767

Prinz, W. (1990). A common coding approach to perception and action. In O. Neumann \& W. Prinz (Eds.), Relationships between perception and action (pp. 167-201). Heidelberg, Berlin: Springer.

Prinz, W. (1997). Perception and action planning. European Journal of Cognitive Psychology, 9, 129-154.

Ramsey, R. (2018). What are reaction time indices of automatic imitation measuring? Consciousness and Cognition, 65, 240254. https://doi.org/10.1016/j.concog.2018.08.006

Reutner, L., \& Wänke, M. (2013). For my own benefit or for the benefit of others: Reminders of money moderate the effects of self-related versus other-related persuasive arguments. Social Psychological and Personality Science, 4, 220-223. https://doi. org/10.1177/1948550612450052
Rohrer, D., Pashler, H., \& Harris, C. (2015). Do subtle reminders of money change people's political views? Journal of Experimental Psychology: General, 144, e73-e85. https://doi.org/10.1037/ xge0000058

Santiesteban, I., Banissy, M. J., Catmur, C., \& Bird, G. (2015). Functional lateralization of temporoparietal junction-imitation inhibition, visual perspective-taking and theory of mind. European Journal of Neuroscience, 42, 2527-2533.

Schneider, W., Eschman, A., \& Zuccolotto, A. (2002). E-Prime: User's guide. Psychology Software Incorporated: Pittsburgh, PA.

Schuler, J., \& Wänke, M. (2016). A fresh look on money priming: Feeling privileged or not makes a difference. Social Psychological and Personality Science, 7, 366-373. https://doi.org/ 10.1177/1948550616628608

Schwarz, N. (1999). Self-reports: How the questions shape the answers. American Psychologist, 54, 93-105. https://doi.org/ 10.1037/0003-066X.54.2.93

Simon, H. A. (1967). Motivational and emotional controls of cognition. Psychological Review, 74, 29-39. https://doi.org/ 10.1037/h0024127

Sowden, S., Koehne, S., Catmur, C., Dziobek, I., \& Bird, G. (2015). Intact automatic imitation and typical spatial compatibility in autism spectrum disorder: Challenging the broken mirror theory. Autism Research, 9, 292-300. https://doi.org/10.1002/ aur. 1511

Spengler, S., Brass, M., Kühn, S., \& Schütz-Bosbach, S. (2010). Minimizing motor mimicry by myself: Self-focus enhances online action-control mechanisms during motor contagion. Consciousness and Cognition, 19, 98-106. https://doi.org/ 10.1016/j.concog.2009.12.014

Stanley, D. J., \& Spence, J. R. (2014). Expectations for replications: Are yours realistic? Perspectives on Psychological Science, $\quad 9, \quad 305-318 . \quad$ https://doi.org/10.1177/ 1745691614528518

Stevens, M., Lammertyn, J., Verbruggen, F., \& Vandierendonck, A. (2006). Tscope: AC library for programming cognitive experiments on the MS Windows platform. Behavior Research Methods, 38, 280-286. https://doi.org/10.3758/BF03192779

Stürmer, B., Aschersleben, G., \& Prinz, W. (2000). Correspondence effects with manual gestures and postures: A study of imitation. Journal of Experimental Psychology: Human Perception and Performance, 26, 1746-1759. https://doi.org/10.1037/ 0096-1523.26.6.1746

Tanner-Smith, E. E., Tipton, E., \& Polanin, J. R. (2016). Handling complex meta-analytic data structures using robust variance estimates: A tutorial in R. Journal of Developmental and LifeCourse Criminology, 2, 85-112.

Teng, F., Chen, Z., Poon, K.-T., Zhang, D., \& Jiang, Y. (2016). Money and relationships: When and why thinking about money leads people to approach others. Organizational Behavior and Human Decision Processes, 137, 58-70.

Vadillo, M. A., Hardwicke, T. E., \& Shanks, D. R. (2016). Selection bias, vote counting, and money-priming effects: A comment on Rohrer, Pashler, and Harris (2015) and Vohs (2015). Journal of Experimental Psychology: General, 145, 655-663. https://doi. org/10.1037/xge0000157

van Baaren, R. B., Maddux, W. W., Chartrand, T. L., De Bouter, C., \& van Knippenberg, A. (2003). It takes two to mimic: Behavioral consequences of self-construals. Journal of Personality and Social Psychology, 84, 1093-1102. https://doi.org/10.1037/ 0022-3514.84.5.1093

Vohs, K. D. (2015). Money priming can change people's thoughts, feelings, motivations, and behaviors: An update on 10 years of experiments. Journal of Experimental Psychology: General, 144 , e86-e93. https://doi.org/10.1111/j.1467-8721.2008.00576.x 
Vohs, K. D., Mead, N. L., \& Goode, M. R. (2006). The psychological consequences of money. Science, 314, 1154-1156. https://doi. org/10.1126/science.1132491

Vohs, K. D., Mead, N. L., \& Goode, M. R. (2008). Merely activating the concept of money changes personal and interpersonal behavior. Current Directions in Psychological Science, 17, 208212. https://doi.org/10.1111/j.1467-8721.2008.00576.x

Wang, Y., \& Hamilton, A. (2013). Understanding the role of the "self" in the social priming of mimicry. PLoS One, 8, e60249.

Wang, Y., \& Hamilton, A. F. d. C. (2015). Anterior medial prefrontal cortex implements social priming of mimicry. Social Cognitive and Affective Neuroscience, 10, 486-493. https://doi.org/ 10.1093/scan/nsu076

Weingarten, E., Chen, Q., McAdams, M., Yi, J., Hepler, J., \& Albarracín, D. (2016). From primed concepts to action: A metaanalysis of the behavioral effects of incidentally presented words. Psychological Bulletin, 142, 472-497. https://doi.org/ 10.1037/bul0000030

Zedelius, C. M., Veling, H., \& Aarts, H. (2013). I was unaware and I needed the money! Success and failure in behavioral regulation toward consciously and unconsciously perceived monetary cues. Social Cognition, 31, 81-93. https://doi.org/10.1521/soco.2013. 31.1.81

Zink, C. F., Pagnoni, G., Martin-Skurski, M. E., Chappelow, J. C., \& Berns, G. S. (2004). Human striatal responses to monetary reward depend on saliency. Neuron, 42, 509-517.

\section{History}

Received August 22, 2016

Revision received December 5, 2019

Accepted December 9, 2019

Published online February 14, 2020

\section{Open Data}

Stimuli and data of both experiments, and the preregistration of the preregistered experiment are made open accessible at the Open Science Framework (OSF) under the following link: https://osf.io/ncvfs/?view_only=82162828bf194135b8bb63b483 ed 2870

\section{Funding}

This work was partially supported by a grant from the German Research Foundation (DFG; grant number 410562468).

\section{Oliver Genschow}

University of Cologne

Social Cognition Center Cologne

Richard-Strauss Str. 2

50931 Köln

Germany

oliver.genschow@uni-koeln.de 